\title{
Plasma exchange for acute on chronic liver failure: is there a light at the end of the tunnel?
}

\author{
Rakhi Maiwall $^{1} \cdot$ Richard Moreau $^{2,3,4,5}$
}

Received: 24 November 2015/Accepted: 8 January 2016/Published online: 4 February 2016

(C) Asian Pacific Association for the Study of the Liver 2016

In the last decade there has been renewed interest in artificial liver support systems (ALSs), which have emerged as a potential tool for management of patients with acute and acute-on-chronic liver failure (ACLF) [1,2]. Traditionally, management of these patients has been based on hemodynamic, neuroprotective and renal support systems with liver transplantation (LT) as the only definitive treatment option [1]. However, LT remains a limited resource, and therefore there is an unmet need for alternative therapies for this disease. Use of ALSs was shown to be associated with a reduction in mortality in controlled and uncontrolled series compared to traditional treatment options in these patients [2]. However, the mechanisms by which ALSs improve outcome of ACLF are poorly understood. The first stage of ACLF is characterized by a systemic inflammatory response syndrome (SIRS), which may be excessive and cause tissue damage. The SIRS stage further progresses to one of compensatory antiinflammatory response syndrome (CARS) or a state of immunoparalysis. This stage is characterized by an impaired ability to clear bacterial pathogens, development of second or nosocomial

Rakhi Maiwall

rakhi_2011@yahoo.co.in

1 Department of Hepatology, Institute of Liver and Biliary Science, New Delhi, India

2 Inserm, U1149, Centre de Recherche sur l'Inflammation (CRI), Clichy and Paris, France

3 UMRS1149, Université Paris Diderot-Paris 7, Paris, France

4 Département Hospitalo-Universitaire (DHU) UNITY, Service d'Hépatologie, Hôpital Beaujon, Assistance Publique-Hôpitaux de Paris, Clichy, France

5 Laboratoire d'Excellence Inflamex, ComUE Sorbonne Paris Cité, Paris, France infections, opportunistic infections and death [1-4]. Defects in the clearance of antibody-coated bacteria and the complement system, a decrease in HLA-DR expression on monocytes and defects in neutrophil function, i.e., impairment of the phagocytic capacity, chemotaxis and an increase in the resting oxidative burst are seen in this phase [4]. Therefore, it may be important to restore an appropriate inflammatory response of the host in the initial phase of SIRS and prevent progression to CARS. Plasma exchange (PE), by causing elimination of a wide array of accumulated toxins in patients with liver failure, can facilitate recovery of the failing liver by providing an environment conducive to liver regeneration and can be used as an effective therapy for bridging the failing liver to LT $[2,5]$. During this procedure, the patient's plasma is removed an exchanged with fresh frozen plasma. Treatment with PE in addition to correction of coagulopathy can potentiate removal of toxic metabolites as well as the poorly identified mediators of multiorgan failure. In a recent prospective, multicenter, randomized controlled trial of 182 patients with acute liver failure, treatment with high-volume PE was shown to modulate the inflammatory cytokine storm, dampen the antiinflammatory responses and ameliorate multiorgan failure, which was associated with improved transplant-free survival [5]. However, there have been no randomized controlled trials of PE for ACLF.

In a retrospective study Wan Yue Meng and colleagues investigated the efficacy of PE in patients with acute-onchronic liver failure and acute decompensation of cirrhosis treated with entecavir (ETV) [6]. Patients who were extremely sick with unstable hemodynamics and disseminated intravascular coagulation were excluded from the study. Patients were enrolled into two groups, i.e., the PE group $(n=38)$ and control group $(n=120)$. All enrolled patients were treated with entecavir $0.5 \mathrm{mg}$ daily along 
with the standard of care. Patients in the PE group received 2-5 sessions of PE therapy. PE therapy was performed twice every week until the patient's condition was stable. Patients in the PE group were sicker with higher MELD scores and lower albumin levels compared to the control group. The proportion of patients with other complications such as hepatic encephalopathy, variceal bleed and renal dysfunction was not different between the two groups at baseline. The baseline virological parameters, such as the presence of $\mathrm{HBeAg}$ as well as HBV DNA, were also not significantly different between the two groups. At 3-month follow-up it was noted that patients in the PE group had lower mortality compared to the control group. The cumulative survival rate at 1 and 3 months in the PE and control group was $37,18,29$ and $14 \%$, respectively (log rank $p=0.001$, HR 1.98, $95 \%$ CI 1.29-3.03). On multivariate analysis, treatment with PE therapy was associated with improved survival at 3 months. Further, this benefit in survival was not associated with improved virological response as no significant difference was noted in the mean HBV DNA in patients treated with PE compared to the control group, $3.69 \pm 0.26$ and $3.81 \pm 0.19 \log 10$ copies/ $\mathrm{ml}$ and $3.42 \pm 0.12$ and $3.54 \pm 0.21 \log 10 \mathrm{copies} / \mathrm{ml}$ at week 2 and 4, respectively. Moreover, the number of patients with $\mathrm{HBeAg}$-positive disease achieving $\mathrm{HBeAg}$ seroconversion was also not significantly different between the two groups.

Even though the study has the limitation of a retrospective and monocentric design, it nevertheless provides useful information on additional therapeutic approaches that could be beneficial in improving liver functions in patients with HBV-related ACLF (HBV-ACLF). Furthermore, despite the fact that patients in the PE group were sicker than those in the control group, a significantly better survival at both 1 and 3 months was seen in the PE group compared to the control group. All patients were treated with entecavir and conventional treatment for the associated complications.

Published data from controlled and uncontrolled series has demonstrated a survival benefit with treatment with nucleos(t)ide analogs in patients with HBV-ACLF related to potent viral suppression [7]. Therefore, according to the current recommendations, all patients with HBV-ACLF should be immediately considered for antiviral therapy to reduce the severity of liver failure and the risk of viral recurrence post-LT [7]. However, unfortunately, only limited benefit has been reported for antivirals alone in HBV-ACLF, necessitating the exploration of alternative therapeutic options for these patients [8]. Other options that have been evaluated in clinical trials include detoxification strategies with artificial liver support systems (ALSs) and therapies to potentiate liver regeneration [8]. None of the currently available ALS devices, i.e., the molecular adsorbent recirculating system (MARS), single-pass albumin dialysis (SPAD) and Prometheus, were associated with a survival benefit in randomized controlled trials done in patients with ACLF [9]. However, data from uncontrolled series have suggested a beneficial role of plasma exchange in these patients because of the clearance of ammonia [10], inflammatory cytokines [11], markers of oxidative stress, i.e., advanced oxidation protein products [12], and amelioration of dysregulated immune systems [13, 14]. In a Chinese study of patients with hepatitis B-related ACLF, it was seen that a decrease in the MELD score after treatment with artificial liver support pre-transplantation led to improved survival post-transplantation, which was comparable to that of patients who underwent emergency liver transplantation [15]. In another study, 62 patients with HBV ACLF who received PE treatment were compared with 131 patients treated with standard of care. The 30-day survival rate of the patients who received PE was significantly higher compared to controls (41.9 versus $25.2 \%$; $p<0.05)$. Interestingly, this benefit was limited only to patients with less severe disease with MELD scores in the range of 20-30 and was not seen for patients with MELD scores $>30$ [16]. Further, a combination of PE treatment with continuous hemodialfiltration [17], plasma bilirubin adsorption [18] or MARS [19] has been shown to be more effective than PE alone. The reason for the lack of a consistent survival benefit with PE and other ALS systems remains the functional incompetence, as these provide only the detoxification function of the entire armamentarium of liver functions. Therefore, therapies that incorporate synthetic functions by means of living hepatocytes in the 'bioartficial liver' or could potentiate hepatic regeneration look more realistic [2]. In patients with HBV-ACLF, randomized controlled trials have shown an improvement in survival by use of mesenchymal stem cells and granulocyte colony-stimulating factor (G-CSF) [20, 21]. A strategy of combining PE with G-CSF has also been proposed; this needs to be studied in larger randomized controlled clinical trials [22].

In summary, even though liver transplant remains the primary treatment modality for patients with HBV ACLF, ALSs including PE can be considered as an effective form of bridging therapy in combination with antiviral therapy in patients with a MELD scores $<30$ and without multiorgan failure. $\mathrm{PE}$ in these patients not only provided transient improvement in biochemical and clinical parameters and help in supporting the failing liver until LT, but could also potentially rescue patients to spontaneous regeneration. In patients with more severe liver failure with a MELD score $>30$, the benefit of PE remained questionable. It would also be interesting to explore the strategy of combining PE with G-CSF or stem cell therapy in patients with HBVACLF. However, considering the limited available 
evidence, larger prospective randomized controlled trials are needed before recommendations can be made for routine incorporation of PE into standard clinical practice.

\section{References}

1. Sarin SK, Kedarisetty CK, Abbas Z, Amarapurkar D, Bihari C, Chan AC, et al. APASL ACLF Working Party. Acute-on-chronic liver failure: consensus recommendations of the Asian Pacific Association for the study of the liver (APASL) 2014. Hepatol Int 2014;8(4):453-471

2. Maiwall R, Maras JS, Nayak SL, Sarin SK. Liver dialysis in acute-on-chronic liver failure: current and future perspectives. Hepatol Int 2014;8(Suppl 2):505-513

3. Moreau R, Jalan R, Arroyo V. Acute-on-chronic liver failure: recent concepts. J Clin Exp Hepatol 2015;5(1):81-85

4. Dirchwolf M, Ruf AE. Role of systemic inflammation in cirrhosis: from pathogenesis to prognosis. World $\mathrm{J}$ Hepatol 2015;7(16):1974-1981

5. Larsen FS, Schmidt LE, Bernsmeier C, Rasmussen A, Isoniemi $\mathrm{H}$, Patel VC, et al. High-volume plasma exchange in patients with acute liver failure: An open randomised controlled trial. J Hepatol 2015;64(1):69-78. doi:10.1016/j.jhep.2015.08.018

6. Yue-Meng W, Yang LH, Yang JH, Xu Y, Yang J, Song GB, et al. The effect of plasma exchange on entecavir-treated chronic hepatitis B patients with hepatic de-compensation and acute-onchronic liver failure. Hepatol Int 2015. [Epub ahead of print] PubMed PMID: 26482576

7. Xie F, Yan L, Lu J, Zheng T, Shi C, Ying J, et al. Effects of nucleoside analogue on patients with chronic hepatitis B-associated liver failure: meta-analysis. PLoS One 2013;8(1):e54773

8. Liu XY, Peng F, Pan YJ, Chen J. Advanced therapeutic strategies for HBV-related acute-on-chronic liver failure. Hepatobiliary Pancreat Dis Int 2015;14(4):354-360

9. Vaid A, Chweich H, Balk EM, Jaber BL. Molecular adsorbent recirculating system as artificial support therapy for liver failure: a meta-analysis. ASAIO J 2012;58(1):51-59

10. Ye JL, Ye B, Lv JX, Mao WL, Gu B. Changes of ammonia levels in patients with acute on chronic liver failure treated by plasma exchange. Hepatogastroenterology 2014;61(129):141-145

11. Mao WL, Chen Y, Chen YM, Li LJ. Changes of serum cytokine levels in patients with acute on chronic liver failure treated by plasma exchange. J Clin Gastroenterol 2011;45(6):551-555

12. Liu H, Han T, Tian J, Zhu ZY, Liu Y, Li Y, Xiao SX, Li Y, Feng YY. Monitoring oxidative stress in acute-on-chronic liver failure by advanced oxidation protein products. Hepatol Res 2012;42(2):171-180

13. Mao WL, Lou YF, Ye B, Lin S, Chen YM, Chen Y. Changes in peripheral CD4 + CD25(high) regulatory $\mathrm{T}$ cells in the acute-onchronic liver failure patientswith plasma exchange treatment. Inflammation 2012;35(2):436-444

14. Yu JW, Sun LJ, Zhao YH, Li SC. Prediction value of model for end-stage liver disease scoring system on prognosis in patients with acute-on-chronic hepatitis B liver failure after plasma exchange and lamivudine treatment. J GastroenterolHepatol 2008;23(8 Pt 1):1242-1249

15. Ling Q, Xu X, Wei Q, Liu X, Guo H, Zhuang L, et al. Downgrading MELD improves the outcomes after liver transplantation in patients with acute-on-chronic hepatitis B liver failure. PLoS One 2012;7(1):e30322

16. Mao W, Ye B, Lin S, Fu Y, Chen Y, Chen Y. Prediction value of model for end-stage liver disease scoring system on prognosis in the acute on chronic liver failure patients with plasma exchange treatment. ASAIO J 2010;56(5):475-478

17. Sadahiro T, Hirasawa H, Oda S, Shiga H, Nakanishi K, Kitamura $\mathrm{N}$, Hirano T. Usefulness of plasma exchange plus continuous hemodiafiltration to reduce adverse effects associated with plasma exchange in patients with acute liver failure. Crit Care Med 2001;29(7):1386-1392

18. Zhou PQ, Zheng SP, Yu M, He SS, Weng ZH. Prognosis of acute-on-chronic liver failure patients treated with artificial liver support system. World J Gastroenterol 2015;21(32):9614-9622

19. Huang YK, Tan DM, Xie YT, Fan XG, Huang Y, Liu ZB, Li SL. Randomized controlled study of plasma exchange combined with molecular adsorbent re-circulating system for the treatment of liver failure complicated with hepatic encephalopathy. Hepatogastroenterology 2012;59(117):1323-1326

20. Duan XZ, Liu FF, Tong JJ, Yang HZ, Chen J, Liu XY, et al. Granulocyte-colony stimulating factor therapy improves survival in patients with hepatitis B virus-associated acute-onchronic liver failure. World J Gastroenterol 2013;19(7):1104-1110

21. Shi M, Zhang Z, Xu R, Lin H, Fu J, Zou Z, et al. Human mesenchymal stem cell transfusion is safe and improves liver function in acute-on-chronic liver failure patients. Stem Cells Transl Med 2012;1(10):725-731

22. Piscaglia AC, Arena V, Passalacqua S, Gasbarrini A. A case of granulocyte-colony stimulating factor/plasmapheresis-induced activation of granulocyte-colony stimulating factor-positive hepatic progenitors in acute-on-chronic liver failure. Hepatology 2015;62(2):649-652 